\title{
Obstruktive Schlaf-Apnoe und Schlaganfall
}

\author{
Obstructive Sleep Apnea and Stroke
}

Autoren

Institute
R. Dumitrascu' ${ }^{1}$, H. Tiede ${ }^{1}$, B. Rosengarten ${ }^{2}$, R. Schulz ${ }^{1}$

${ }^{1}$ Medizinische Klinik II, Universitätsklinik Gießen und Marburg, Standort Gießen

${ }^{2}$ Klinik für Neurologie, Universitätsklinik Gießen und Marburg, Standort Gießen

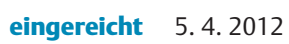
akzeptiert nach Revision 23. 5.2012

Bibliografie

DOI http://dx.doi.org/ 10.1055/s-0032-1309966 Pneumologie 2012; 66: 476-479 (c) Georg Thieme Verlag KG Stuttgart · New York ISSN 0934-8387

Korrespondenzadresse: Prof. Dr. Richard Schulz Universitätsklinik Gießen und Marburg

Medizinische Klinik II

Klinikstr. 33

35392 Gießen

Richard.Schulz@innere.med.unigiessen.de

Serienherausgeber: H. W. Duchna, Wangen W. Randerath, Solingen

\section{Zusammenfassung \\ $\nabla$}

Mehr als die Hälfte der hospitalisierten Schlaganfall-Patienten leiden an schlafbezogenen Atmungsstörungen und $5-10 \%$ der erstdiagnostizierten Patienten mit obstruktiver Schlaf-Apnoe (OSA) haben einen Schlaganfall in der Anamnese. Epidemiologische Studien haben gezeigt, dass die unbehandelte OSA ein unabhängiger Risikofaktor für einen Schlaganfall ist. Pathophysiologisch spielen wahrscheinlich mehrere Faktoren eine Rolle (OSA-assoziierte arterielle Hypertonie, Atherosklerose, Vorhofflimmern, paradoxe Embolie durch ein offenes Foramen ovale und gestörte zerebrovaskuläre Reaktivität). Eine koexistente OSA verschlechtert den funktionellen Outcome und das Überleben von Patienten mit Schlaganfall. Andererseits hat die CPAP-Therapie günstige Effekte auf das Neuauftreten und die Rezidivrate von Schlaganfällen bei OSA und auch auf den klinischen Verlauf von Patienten, die einen Schlaganfall erlitten haben.

\section{Einführung \\ $\nabla$}

Der Schlaganfall ist ein Notfall, bei dem sofort medizinische Hilfe erforderlich ist und eine Krankenhauseinweisung erfolgen sollte. In Deutschland geht man jährlich von ca. 160000 Schlaganfällen aus. Die Erkrankung ist die dritthäufigste Todesursache und der häufigste Grund für eine bleibende mittlere bis schwere Behinderung [1,2].

Indikatoren eines Schlaganfalls sind plötzlich auftretende neurologische Ausfälle wie sensorische oder motorische Defizite, Sprech- und Sprachstörungen sowie Sehstörungen. Im Vordergrund stehende Zeichen einer Vigilanz-, Atem- oder Kreislaufstörung oder Schwindel deuten auf eine Hirnstammischämie hin. Mithilfe bildgebender Verfahren können Blutungen, früher als hämorrhagischer Schlaganfall bezeichnet, als Ursache ausgeschlossen werden. Beim ischämischen

\section{Abstract \\ $\nabla$}

More than half of the patients hospitalised with stroke suffer from sleep-disordered breathing and $5-10 \%$ of patients with newly diagnosed obstructive sleep apnea (OSA) have a history of stroke. Epidemiological studies have shown that untreated OSA is an independent risk factor for stroke. Various pathophysiological mechanisms may contribute to the development of stroke in these patients (i.e., OSA-associated arterial hypertension, atherosclerosis, atrial fibrillation, paradoxical embolism through a patent foramen ovale and disturbed cerebrovascular reactivity). Coexistent OSA has a negative impact on both the recovery of neurological functions and the survival of patients with stroke. On the other hand, CPAP therapy has beneficial effects on the incidence and recurrence of stroke in OSA and also on the clinical outcome of those patients who are victims of stroke.

Schlaganfall besteht eine Minderdurchblutung infolge einer Atherosklerose der hirnversorgenden Arterien oder einer Thromboembolie in das Gehirn. Bei der seltener vorkommenden Hirnblutung ist die Ursache eine Zerreißung kleiner Gefäße, z.B. im Rahmen einer hypertensiven Blutdruckentgleisung. Die wichtigsten Risikofaktoren des Schlaganfalls sind arterielle Hypertonie, Diabetes mellitus, Nikotinabusus, Bewegungsmangel und Adipositas [3].

Das Ziel dieses kurz gefassten Übersichtsartikels ist es, den aktuellen Wissensstand über die $\mathrm{Zu}$ sammenhänge zwischen obstruktiver Schlaf-Apnoe (OSA) und Schlaganfall darzustellen. Hierbei sollen epidemiologische, pathophysiologische, klinische und therapeutische Aspekte Erwähnung finden. 


\section{Epidemiologie}

\section{$\nabla$}

Schlafbezogene Atmungsstörungen (SBAS) sind im Anschluss an die Akutphase eines Schlaganfalls sehr häufig anzutreffen. Eine Metaanalyse beschreibt eine mittlere Prävalenz von $>50 \%$ bei diesen Patienten ([4], $\odot$ Abb. 1). Zu berücksichtigen ist allerdings, dass in den betreffenden Studien nicht immer eine Differenzierung zwischen OSA und zentraler Schlaf-Apnoe (ZSA) erfolgte. So kann sich eine ZSA als Folge des Schlaganfalls im weiteren zeitlichen Verlauf wieder zurückbilden, während die OSA in der Regel schon vor dem Schlaganfall vorhanden war und als ursächlich für diesen angesehen werden kann [5].

Bei Erstdiagnose einer OSA haben 5-10\% dieser Patienten bereits einen Schlaganfall erlitten, wie z.B. auch unsere Arbeitsgruppe in einer retrospektiv durchgeführten Untersuchung finden konnte [6]. Dies bedeutet im Vergleich zur Allgemeinbevölkerung eine in etwa um den Faktor 5 gesteigerte Prävalenz des Schlaganfalls bei OSA.

Verschiedene epidemiologische Studien wie die Wisconsin Sleep Cohort Study und die Sleep Heart Health Study haben gefunden, dass die OSA ein eigenständiger Risikofaktor für einen Schlaganfall ist und die oben genannten klassischen Risikofaktoren um die Berücksichtigung einer OSA erweitert werden müssen. Dabei ist die Höhe des Risikos vom Schweregrad der OSA abhängig. Dieses steigt schon bei Vorliegen einer moderaten OSA (Apnoe-Hypopnoe-Index $[\mathrm{AHI}] \geq 15 / \mathrm{h}$ ) an und erreicht bei Patienten mit einem $\mathrm{AHI} \geq 30 / \mathrm{h}$ relativ hohe adjusted odds bzw. hazard ratios von circa 3 [7-9].

\section{Pathophysiologie \\ $\nabla$}

Mehrere Faktoren können zur Entstehung von Schlaganfällen bei OSA-Patienten beitragen. Am wichtigsten ist in diesem Zusammenhang wohl die OSA-assoziierte arterielle Hypertonie mit dem charakteristischen non-dipping der Blutdruckwerte während des Schlafes. Ein weiterer pathophysiologischer Mechanismus könnte die Begünstigung der Atherosklerose der hirnversorgenden Arterien infolge der OSA-assoziierten nächtlichen Hypoxämie sein. Hierfür sprechen Studien, welche die IntimaMedia-Dicke der A. carotis mittels hochauflösender Sonografie gemessen haben ([10], A Abb.2). Das mit der OSA assoziierte Schnarchen kann möglicherweise über ein Vibrationstrauma der Gefäßwand ebenfalls zur Entwicklung einer Atherosklerose beitragen, die sich vornehmlich an den Halsgefäßen manifestiert [11].

Weiterhin könnte das Auftreten embolisch bedingter Schlaganfälle durch die OSA begünstigt werden. Zum einen kann die unbehandelte OSA Vorhofflimmern triggern [12], welches dann via Thrombenbildung im linken Vorhof und nachfolgender Embolisierung in das Gehirn einen Schlaganfall bewirkt. Zum anderen ist es denkbar, dass es bei Vorliegen einer tiefen Beinvenenthrombose und gleichzeitiger OSA durch die vermehrten intrathorakalen Druckschwankungen zu einer Translokation von Thromben durch ein offenes Foramen ovale in das zentrale Nervensystem kommt (sog. paradoxe Embolie [13]).

Schließlich ist die Vasoreaktivität der zerebralen Gefäße in Antwort auf verschiedene Stimuli bei Patienten mit OSA eingeschränkt [14,15], wodurch es zu Minderungen des Perfusionsdrucks und in der Folge zu Schlaganfällen kommen könnte.

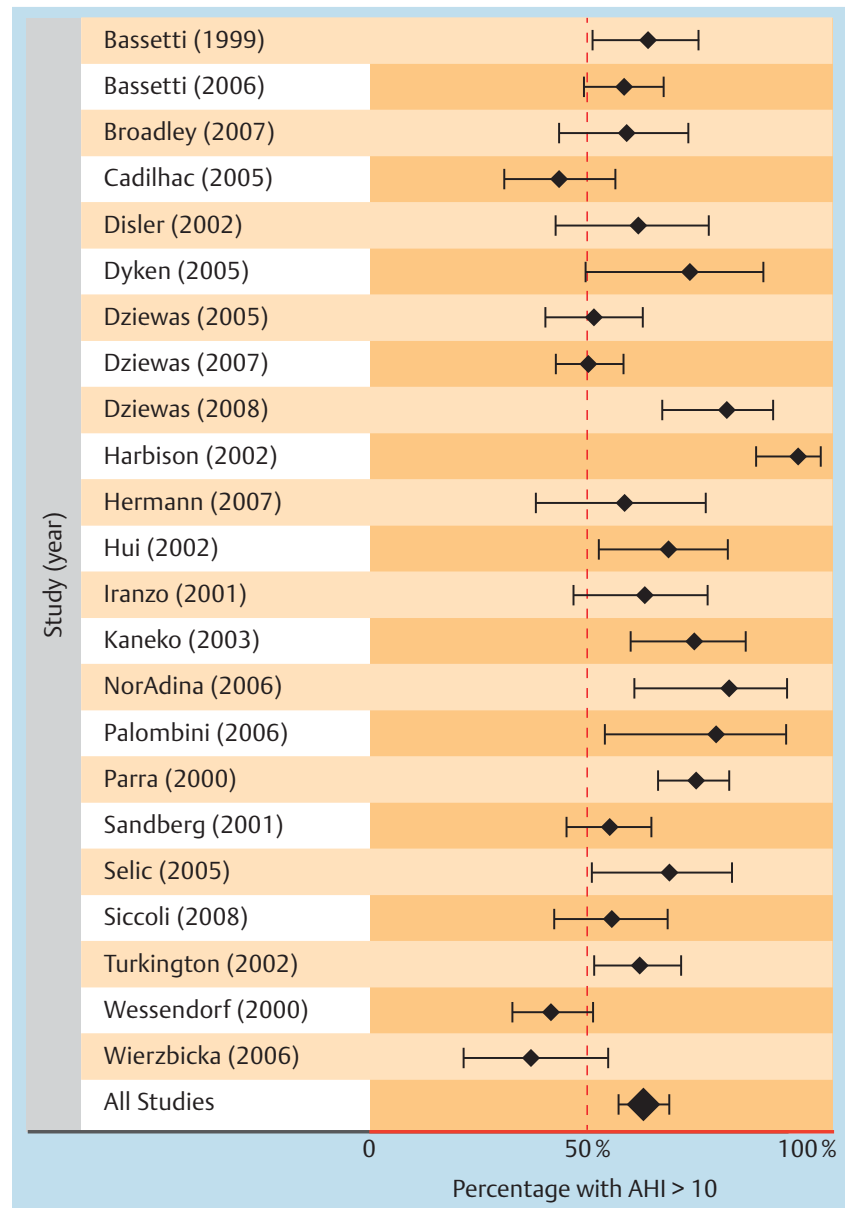

Abb. 1 Metaanalyse zur Prävalenz schlafbezogener Atmungsstörungen bei mit Schlaganfall hospitalisierten Patienten (aus Referenz [4]).

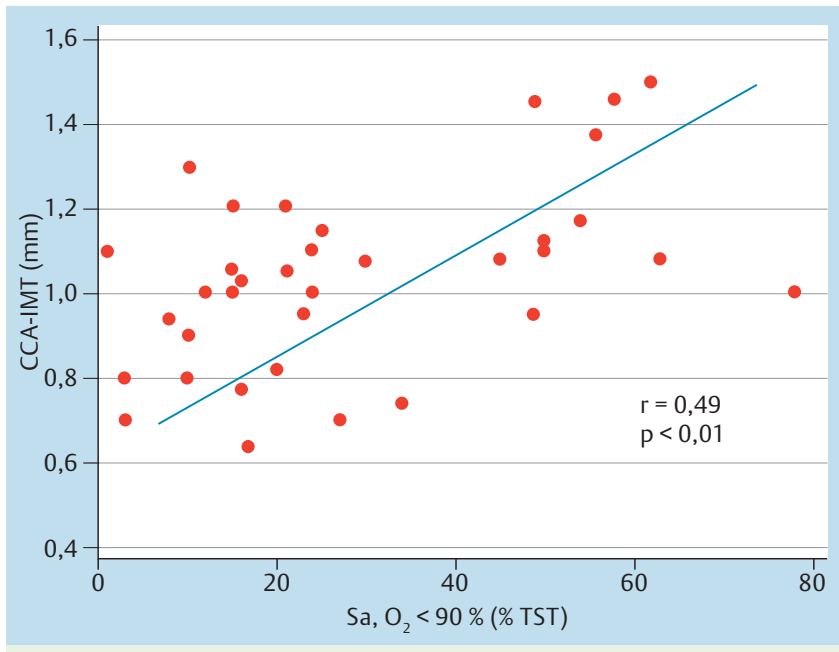

Abb. 2 Intima-Media-Dicke der A. carotis communis in Abhängigkeit vom Ausmaß der nächtlichen Hypoxie bei OSA (modifiziert nach Referenz [10]). Abdruck mit freundlicher Genehmigung der ERS. 


\section{Klinik}

\section{$\nabla$}

OSA-assoziierte Schlaganfälle treten bevorzugt in den frühen Morgenstunden bzw. beim/nach dem Aufwachen auf, ihre klinische Symptomatik unterscheidet sich aber nicht von denjenigen Schlaganfällen, die sich zu anderen Tageszeiten ereignen [16]. Zu beachten ist, dass die betroffenen Patienten weniger unter Tagesschläfrigkeit leiden und einen niedrigeren Body-Mass-Index haben als OSA-Patienten ohne Apoplex [17]. Dies kann möglicherweise dazu beitragen, dass die Diagnose der OSA nach abgelaufenem Schlaganfall nicht oder erst mit zeitlicher Verzögerung gestellt wird.

Das gleichzeitige Bestehen einer OSA hat ungünstige Auswirkungen auf den klinischen Verlauf eines Schlaganfalls. So können sich bedingt durch die OSA neurologische Funktionen nur verzögert oder überhaupt nicht mehr erholen [18,19]. Nachgewiesen ist auch, dass Schlaganfall-Patienten mit unbehandelter OSA eine höhere Mortalität aufweisen [20,21] ( Abb.3).

\section{Therapie \\ $\nabla$}

Mit der CPAP-Therapie steht eine effektive Behandlung der OSA zur Verfügung. In Bezug auf das Thema dieses Review-Artikels ist hierbei zu unterscheiden zwischen der CPAP-Therapie von OSA-Patienten ohne und mit Zustand nach Schlaganfall. Beobachtungsstudien zeigen, dass bei OSA-Patienten, die bisher noch keinen Apoplex erlitten haben, die Inzidenz von Schlaganfällen durch CPAP reduziert wird [22].

Randomisierte, placebokontrollierte Studien fehlen in diesem Kontext noch, sind allerdings aus ethischer Sicht auch nicht vertretbar. Insofern sollte unserer Meinung nach bei OSA-Patienten ab einem AHI $\geq 30 / h$ unabhängig vom Vorhandensein einer Tagessymptomatik eine CPAP-Therapie eingeleitet werden, um u.a. auch das Schlaganfall-Risiko zu senken. Berücksichtigt man die Ergebnisse der eingangs genannten epidemiologischen Studien, kann diese Therapieempfehlung möglicherweise schon für Patienten mit moderater OSA (AHI $15-30 / \mathrm{h}$ ) gelten.

OSA-Patienten mit Zustand nach Schlaganfall können ebenfalls von einer CPAP-Therapie profitieren. So können durch die CPAPTherapie zum einen Schlaganfall-Rezidive verhindert werden [23], zum anderen kann die Erholung neurologischer Defizite gefördert werden [24-26]. Beschrieben sind schließlich günstige Effekte auf die Mortalität dieser Patienten [27]. Anzumerken ist in diesem Zusammenhang jedoch, dass die Compliance der CPAP-Therapie bei OSA-Patienten mit bereits abgelaufenem Schlaganfall oft nicht befriedigend ist [28,29]. In dieser Situation sind die transnasale Insufflation und die Vermeidung der Rückenlage u.U. besser praktikable therapeutische Alternativen, obwohl sie eine niedrigere Effektivität als die CPAP-Therapie besitzen $[30,31]$.

\section{Schlussfolgerung}

$\nabla$

OSA und Schlaganfall sind signifikant miteinander assoziiert und die unbehandelte OSA ist ein unabhängiger Risikofaktor für den Schlaganfall. Mehrere pathophysiologische Faktoren tragen zur Entstehung von Schlaganfällen bei OSA bei (OSA-assoziierte arterielle Hypertonie, Atherosklerose, Vorhofflimmern, paradoxe Embolie und gestörte Vasoregulation).

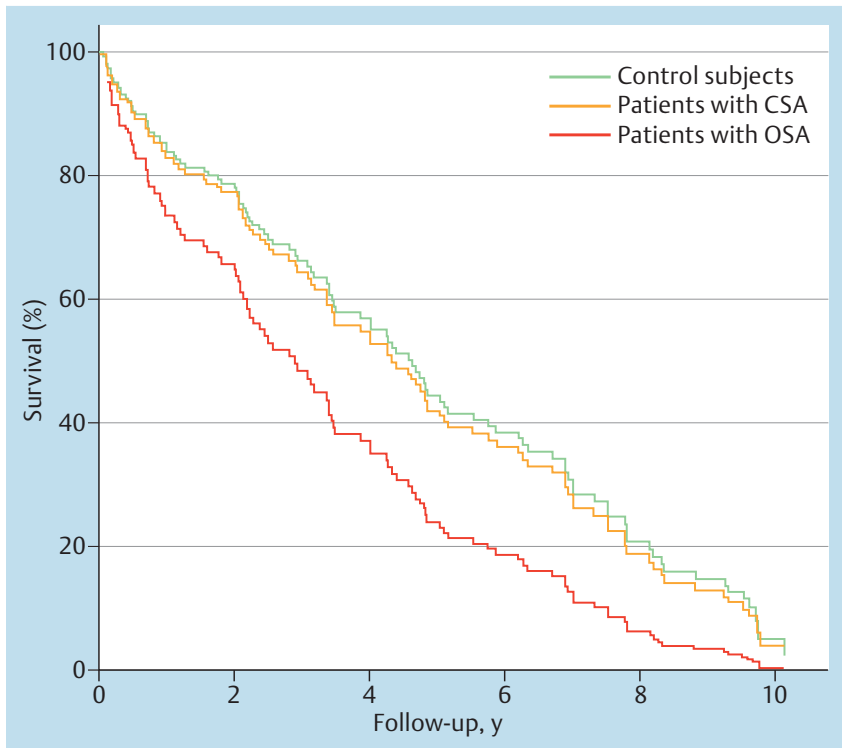

Abb.3 Überlebensraten von Schlaganfall-Patienten ohne Schlaf-Apnoe ("control subjects“) sowie solchen mit zentraler und obstruktiver SchlafApnoe (aus Referenz [21]).

Eine koexistente OSA hat ungünstige Auswirkungen auf den funktionellen Outcome und das Überleben von Patienten mit Schlaganfall. Die CPAP-Therapie kann sowohl das Auftreten von Schlaganfällen als auch die negativen klinischen Konsequenzen bereits abgelaufener Schlaganfälle bei OSA-Patienten vermindern. Im Hinblick auf praktische Durchführbarkeit und Compliance ist die Indikation zur CPAP-Therapie bei OSA-Patienten mit Zustand nach Schlaganfall jedoch individuell zu stellen.

\section{Interessenkonflikt}

Die Autoren geben an, dass kein Interessenkonflikt besteht.

\section{Literatur}

1 http://www.kompetenznetz-schlaganfall.de

2 http://www.destatis.de

3 O'Donnell MJ, Xavier D, Liu L et al. Risk factors for ischaemic and intracerebral haemorrhagic stroke in 22 countries (the INTERSTROKE study): a case-control study. Lancet 2010; 376: 112-123

4 Johnson KG, Johnson DC. Frequency of sleep apnea in stroke and TIA patients: a metaanalysis. J Clin Sleep Med 2010; 6: 131-137

5 Parra O, Arboix A, Bechich $S$ et al. Time course of sleep-related breathing disorders in first-ever stroke or transient ischemic attack. Am J Respir Crit Care Med 2000; 161: 375 - 380

6 Schulz R, Olschewski H, Grimminger $F$ et al. Prävalenz von Schlaganfall und transitorischer ischämischer Attacke (TIA) bei obstruktiver SchlafApnoe: eine retrospektive Erhebung an 187 konsekutiven Patienten. Pneumologie 2000; 54: 575-579

7 Yaggi HK, Concato J, Kernan WN et al. Obstructive sleep apnea as a risk factor for stroke and death. N Engl J Med 2005; 353: 2034 - 2041

8 Arzt M, Young T, Finn $L$ et al. Association of sleep-disordered breathing and the occurrence of stroke. Am J Respir Crit Care Med 2005; 172: $1447-1451$

9 Redline S, Yenokyan G, Gottlieb DJ et al. Obstructive sleep apnea-hypopnea and incident stroke: the sleep heart health study. Am J Respir Crit Care Med 2010; 182: 269-277

10 Schulz R, Seeger W, Fegbeutel C et al. Changes in extracranial arteries in obstructive sleep apnoea. Eur Respir J 2005; 25: 69-74

11 Lee $S A$, Amis TC, Byth $K$ et al. Heavy snoring as a cause of carotid artery atherosclerosis. Sleep 2008; 31: 1207-1213

12 Schulz R, Eisele HJ, Seeger W. Nocturnal atrial fibrillation in a patient with obstructive sleep apnoea. Thorax 2005; 60: 174 
13 Beelke $M$, Angeli S, Del Sette $M$ et al. Obstructive sleep apnea can be provocative for right-to-left shunting through a patent foramen ovale. Sleep 2002; 25: $21-27$

14 Diomedi M, Placidi F, Cupini LM et al. Cerebral hemodynamic changes in sleep apnea syndrome and effect of continuous positive airway pressure treatment. Neurology 1998; 51: 1051 - 1056

15 Foster GE, Hanly PJ, Ostrowski M et al. Effects of continuous positive airway pressure on cerebral vascular response to hypoxia in patients with obstructive sleep apnea. Am J Respir Crit Care Med 2007; 175: 720 725

16 Hsieh SW, Lai CL, Liu CK et al. Obstructive sleep apnea linked to wakeup strokes. J Neurol 2012: (Epub ahead of print)

17 Arzt M, Young T, Peppard PE et al. Dissociation of obstructive sleep apnea from hypersomnolence and obesity in patients with stroke. Stroke 2010; 41: 129-134

18 Good DC, Henkle JQ Gelber D et al. Sleep-disordered breathing and poor functional outcome after stroke. Stroke 1996; 27: 252 - 259

19 Kaneko Y, Hajek VE, Zivanovic $V$ et al. Relationship of sleep apnea to functional capacity and length of hospitalization following stroke. Sleep 2003; 26: 293-297

20 Parra O, Arboix A, Montserrat JM et al. Sleep-related breathing disorders: impact on mortality of cerebrovascular disease. Eur Respir J 2004; 24: 267-272

21 Sahlin C, Sandberg O, Gustafson Yet al. Obstructive sleep apnea is a risk factor for death in patients with stroke. Arch Intern Med 2008; 168: 297-301

22 Marin JM, Carrizo SJ, Vicente E et al. Long-term cardiovascular outcomes in men with obstructive sleep apnoea-hypopnoea with or without treatment with continuous positive airway pressure: an observational study. Lancet 2005; 365: 1046-1053
23 Martinez-Garcia MA, Galiano-Blancart R, Roman-Sanchez P et al. Continuous positive airway pressure treatment in sleep apnea prevents new vascular events after ischemic stroke. Chest 2005; 128: 2123 2129

24 Ryan CM, Bavlev $M$, Green $R$ et al. Influence of continuous positive airway pressure on outcomes of rehabilitation in stroke patients with obstructive sleep apnea. Stroke 2011; 42: 1062 - 1067

25 Parra 0 , Sánchez-Armengol A, Bonnin $M$ et al. Early treatment of obstructive apnoea and stroke outcome: a randomised controlled trial. Eur Respir J 2011; 37: 1128 - 1136

26 Bravata DM, Concato J, Fried T et al. Continuous positive airway pressure: evaluation of a novel therapy for patients with acute ischemic stroke. Sleep 2011; 34: 1271 - 1277

27 Martínez-García MA, Soler-Cataluña JJ, Ejarque-Martínez L et al. Continuous positive airway pressure treatment reduces mortality in patients with ischemic stroke and obstructive sleep apnea: a 5-year follow-up study. Am J Respir Crit Care Med 2009; 180: 36-41

28 Sandberg 0 , Franklin KA, Bucht $G$ et al. Nasal continuous positive airway pressure in stroke patients with sleep apnoea: a randomized treatment study. Eur Respir J 2001; 18: 630-634

29 Hsu CY, Vennelle M, Li HY et al. Sleep-disordered breathing after stroke: a randomised controlled trial of continuous positive airway pressure. ] Neurol Neurosurg Psychiatry 2006; 77: 1143 - 1149

30 Haba-Rubio J, Andries D, Rey V et al. Effect of transnasal insufflation on sleep disordered breathing in acute stroke: a preliminary study. Sleep Breath 2011 [Epub ahead of print]

31 Svatikova A, Chervin RD, Wing JJ et al. Positional therapy in ischemic stroke patients with obstructive sleep apnea. Sleep Med 2011; 12: $262-266$

\section{Erratum}

A. Nienhaus et al. Prävalenz der latenten Tuberkulose-Infektion bei Beschäftigten im Gesundheitswesen Ein Dreiländervergleich. Pneumologie 2011; 65: 726-729

In der Autorenliste wurde der Name eines Co-Autors falsch wiedergegeben: Richtig muss es heißen: D. Tripodi. 